\title{
Full-State-Constrained Adaptive Control for a Class of UAVs Suffering from Coupled Uncertainties Using the HOBLF
}

\author{
Xiaofei Chang, ${ }^{1}$ Kexuan Wang $\mathbb{D},{ }^{2}$ Kang Chen $\mathbb{D},{ }^{1}$ and Wenxing Fu $\mathbb{D}^{1}$ \\ ${ }^{1}$ Unmanned System Research Institute, Northwestern Polytechnical University, Xi'an 710072, China \\ ${ }^{2}$ College of Astronautics, Northwestern Polytechnical University, Xi'an 710072, China \\ Correspondence should be addressed to Kang Chen; mars_legend@163.com
}

Received 9 March 2021; Revised 3 September 2021; Accepted 11 September 2021; Published 11 October 2021

Academic Editor: Jose Vicente Salcedo

Copyright (C) 2021 Xiaofei Chang et al. This is an open access article distributed under the Creative Commons Attribution License, which permits unrestricted use, distribution, and reproduction in any medium, provided the original work is properly cited.

Nowadays, the practical tasks of UAVs are becoming more and more complicated and diversified. In the practical flight process, the large-scale changes of the flight environment, the modeling errors, and the external disturbances may induce the instability of the UAV flight system. Meanwhile, the constraints of the UAV attitudes also have to be guaranteed during the flight process. However, most existing control methods still have limitations in handling the constraints and the multisource disturbances simultaneously. To address this problem, in this paper, we focus on the actual output tracking control for the UAV systems with full-state constraints and multisource disturbances. Firstly, a high-order tan-type barrier Lyapunov function (HOBLF) has been constructed for the UAV to maintain the full-state constraints. Secondly, by combining the adaptive backstepping technique and the fuzzy logic systems, the modeling errors and the unknown nonlinearities of the UAV attitude control system can be handled. Moreover, by properly constructing several adaptive laws, the time-varying disturbances existing in the UAV attitude control system can be suppressed. Finally, the full-state-constrained antidisturbance controller is formed, ensuring that the tracking error approaches arbitrarily to small neighborhood and does not violate the given constraints. The simulation results illustrate the feasibility and the advantages of the proposed method.

\section{Introduction}

Unmanned aerial vehicles (UAVs) have attracted research interest on account of their simple structures, low price, high mobility, and high flexibility. Due to their significant advantages, they have broad application prospects in military [1] and civilian [2]. However, the nonlinear characteristics and strong coupling of UAVs increase the difficulty of UAV control [3]. The aerodynamic parameter perturbations, modeling errors, and external interference caused by changes in flight conditions may cause instability of the flight system or even failure [4].

In order to tackle the control problem of UAVs [5], several approaches have been proposed, such as robust control [6], adaptive control [7], neural networks [8], fuzzy control [9], sliding mode control [10-12], genetic algorithms [13], and linear parameter-varying (LPV) method [14]. Recently, various advanced control methods have been presented for the attitude control of the UAVs. In [15], a nonlinear model predictive control (NMPC) is used to design a high-level controller for a fixed-wing unmanned aerial vehicle. In [16], this method has been improved, and it improved the optimality of the MPC controllers. In [17], $\mathrm{H}$-loop-shaping method is used to provide the stabilizer mode while successfully improving the robustness of the UAV. The LPV model reference method [14] has been further developed in [18] to achieve agile and high-performance tracking objectives of UAVs. Chen et al. [19] used a disturbance observer-based control (DOBC) scheme with transient performance design for UAVs to tackle the mismatched disturbance problem. Besides the disturbance observer, another effective way to account for the unknown dynamics in the UAV is employing active disturbance rejection control (ADRC) [20]. Moreover, by a combination of backstepping and ADRC, an integrated controller is devised for the quadrotor UAV with multiple uncertainties in [21]. 
Based on filtered dynamic inversion, the controller in [22] enabled the fixed-wing UAV to achieve effective altitude command following in the presence of turbulent wind. In [23], an adaptive second-order sliding mode control based on an extended observer is proposed for UAVs to improve performance under different operating conditions. To achieve an ideal convergence performance of the UAV, prescribed performance controllers [24] and finite-time stable controllers [25-27] were designed.

Due to the limitations of actual physical devices, system performance requirements, and safety requirements, most practical systems will have constraints on the input, state, and output, such as nonuniform gantry crane [28], flexible crane systems [29], and robotic manipulator systems [30]. A series of constraint control methods have been proposed, including reference governors [31], model predictive control [32], extremum-seeking control [33], and the notion of set invariance [34, 35]. In recent years, BLF-based constraint control methods have been widely used to deal with state constraints and output constraints because they do not require precise solutions of the system. The synthesis of the barrier Lyapunov function (BLF) with the constraint function is proposed firstly in dealing with the Brunovsky constraint system [36]. Since then, many scholars have focused on studying the adaptive control for uncertain nonlinear systems based on the barrier Lyapunov function. In [37], by the combination of the backstepping method and BLF, an adaptive constrained controller was proposed for partialstate-constrained systems. In [38, 39], nonlinear purefeedback systems and stochastic systems with full-state constraints have been investigated by constructing barrier Lyapunov functions (BLFs). For the MIMO full-stateconstrained nonlinear systems, the opportune integral BLFs were established to prevent violation of the state constraints in [40]. Tee et al. [41] extended the constant constraint in [41] to the time-varying constraint. Furthermore, BLF has also been successfully applied in practical systems, such as electrostatic microactuator [42] and HSV [43, 44]. In [45], a higher-order barrier Lyapunov function (HOBLF) has been firstly proposed while achieving constraint satisfaction without solving the displayed solution of the system, especially for the higherorder uncertain nonlinear system. Given the influence of the external aerodynamic change and strong interference on the UAV in the flight environment, the attitude control of the UAV becomes an important content related to its performance. Therefore, certain state constraints should be applied to its three attitude angles to avoid the occurrence of large deviations. The high-order barrier Lyapunov function can just solve the problem of UAV attitude constraints. Therefore, this paper introduces [45] to solve the problem of UAV state constant constraints. And as far as the authors know, there is no relevant literature that has studied the state constraints of UAVs.

In spite of the process, the HOBLF-based full-stateconstrained antidisturbance control has never been investigated for the UAVs. According to Sun et al. [45], using the HOBLF can achieve practical output tracking control for a category of high-order uncertain nonlinear systems with full-state constraints. Although Tan and Guo [24] considered the full-state-constrained control for the UAVs, the HOBLF has never been utilized. Moreover, the influence of external aerodynamic changes and strong disturbances of UAVs has to be taken into consideration.

Based on the above observations, for UAV systems with full-state constraints,

(i) This paper introduces a high-order tan-type BLF and applies it to the UAV system, whose three attitude angles have state constraints. In addition, when constraints disappear or the high-order system is reduced to a traditional nonlinear system in the form of strict feedback, this BLF is still effective.

(ii) For the time-varying interference that the UAV may encounter during the flight, an adaptive law is constructed to enable the UAV to track the desired signal stably even under interference and has a strong adaptability.

(iii) Compared with the traditional adaptive, DOBC, and general BLF [36], computational burden has been decreased, and the proposed control method has a better tracking effect on account of the HOBLF and fuzzy system.

This paper is organized as follows. In Section 2, the problem formulation and related knowledge are given, while in Section 3, the high-order tan-type BLF, the design procedures, the controllers, and the adaptive law are designed. Section 4 presents the simulation results and comparison, and Section 5 concludes this paper.

\section{Problem Formulation and Related Knowledge}

2.1. System Model. In this paper, we use the nonlinear dynamics of the UAV built in [46]; the details are shown as follows:

$$
\begin{aligned}
\dot{\vartheta} & =\left(\omega_{y} \sin \gamma+\cos \gamma \omega_{z}\right)+d_{\vartheta}, \\
\dot{\psi} & =\frac{\left(\omega_{y} \cos \gamma-\omega_{z} \sin \gamma\right)}{\cos \vartheta}+d_{\psi}, \\
\dot{\gamma} & =\omega_{x}-\tan \vartheta\left(\omega_{y} \cos \gamma-\omega_{z} \sin \gamma\right)+d_{\gamma}, \\
\dot{\Omega}_{I} & =(I)^{-1}\left[M-\Omega_{I} \times\left(I * \Omega_{I}\right)\right]+d_{\Omega_{I}},
\end{aligned}
$$

where $\vartheta, \psi$, and $\gamma$, respectively, represent the pitching, yaw, and rolling attitude angle of the UAV relative to the inertial coordinate system. $\omega_{x}, \omega_{y}$, and $\omega_{z}$ represent the angular velocity of each degree of freedom, respectively, where $\Omega_{I}=\left(\omega_{x}, \omega_{y}, \omega_{z}\right)^{T} . I$ is the rotational inertia matrix of the UAV. $d_{i}\left(i=\theta, \psi, \gamma, \Omega_{I}\right)$ are unknown unmodeled dynamics of the plant. The control scheme is depicted in Figure 1, and the details and expressions of $I$ and $M$ are shown as follows: 


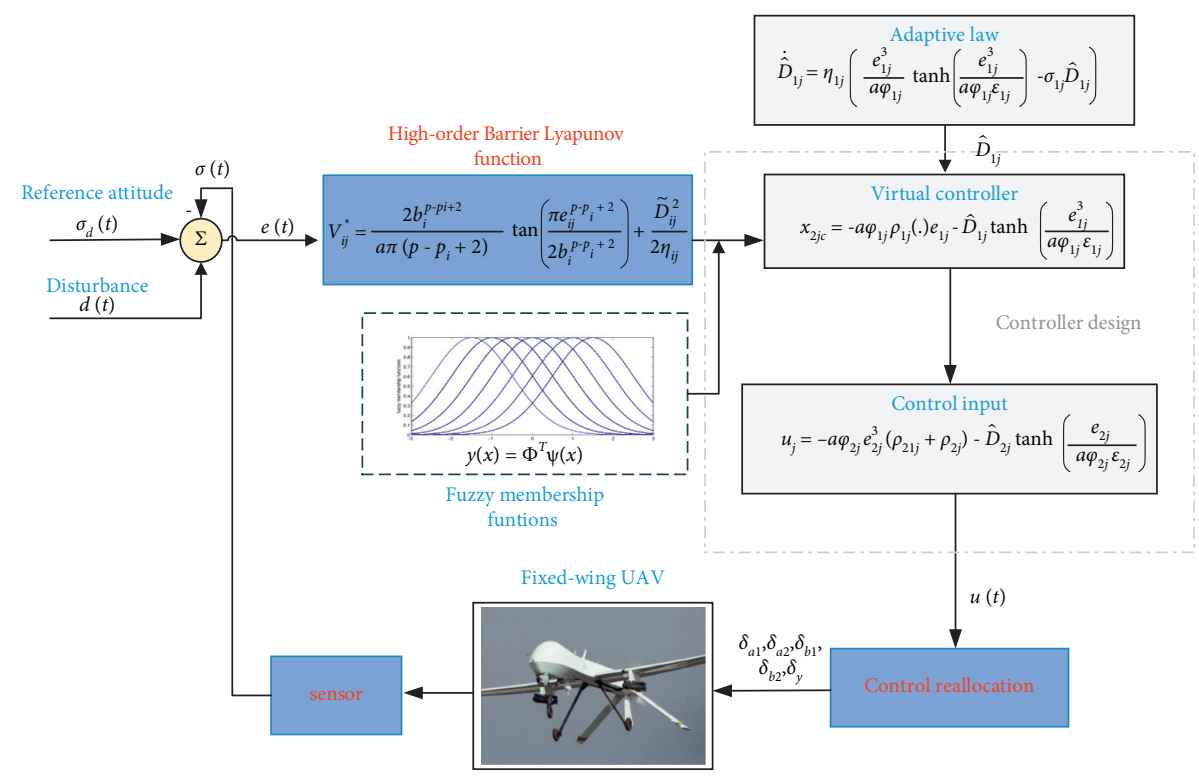

FIgURE 1: The structure of the proposed HOBLF control algorithm.

$$
\begin{aligned}
I & =\left(\begin{array}{ccc}
I_{x x} & 0 & I_{x z} \\
0 & I_{y y} & 0 \\
I_{z x} & 0 & I_{z z}
\end{array}\right), \\
M & =\left(\begin{array}{l}
\bar{q} S b C_{x} \\
\bar{q} S \bar{c} C_{y} \\
\bar{q} S b C_{z}
\end{array}\right),
\end{aligned}
$$

$I_{\Delta \Delta}$ is the product of inertia in both directions, $\bar{q}=\rho V_{T}^{2} / 2$ is the dynamic pressure of air, $V_{T}$ is the total airspeed of the $\mathrm{UAV}, \rho$ is the air density, $S$ is the total area of the wing, $b$ is the wingspan, and $\bar{c}$ is the average chord length. The rolling, pitching, and yaw moments of the UAV are mainly controlled by $C_{x}, C_{y}$, and $C_{z}$, which can be given by

$$
\left\{\begin{array}{l}
C_{x}=C_{x}^{\delta_{a 1}} \delta_{a 1}+C_{x}^{\delta_{a 2}} \delta_{a 2}+C_{x}^{\delta_{b 1}} \delta_{b 1}+C_{x}^{\delta_{b 2}} \delta_{b 2}+C_{x}^{\beta} \beta+\Delta_{x}, \\
C_{y}=C_{y}^{\delta_{y}} \delta_{y}+C_{y}^{\beta} \beta+\Delta_{y}, \\
C_{z}=C_{z 0}+C_{z}^{\delta_{a 1}} \delta_{a 1}+C_{x}^{\delta_{a 2}} \delta_{a 2}+C_{z}^{\delta_{b 1}} \delta_{b 1}+C_{z}^{\delta_{b 2}} \delta_{b 2}+C_{z}^{\alpha} \alpha+\Delta_{z}
\end{array}\right.
$$

where $\delta_{a 1}, \delta_{a 2}$ are the right and left aileron rudder deviation. $\delta_{b 1}, \delta_{b 2}$ are the deviation of the left and right lifter rudder; $\delta_{v}$ is the direction of the wing rudder deviation; $C_{x}$ is the drag coefficient with $C_{x}^{i}\left(i=\delta_{a 1}, \delta_{a 2}, \delta_{b 1}, \delta_{b 2}, \beta\right)$ being the coefficient of $i$ to $C_{x} ; C_{v}$ is the drag coefficient with $C_{y}^{i}\left(i=\delta_{y}, \beta\right)$ being the coefficient of $i$ to $C_{v} ; C_{z}$ is the drag coefficient with $C_{z}^{i}\left(i=\delta_{a 1}, \delta_{a 2}, \delta_{b 1}, \delta_{b 2}, \alpha\right)$ being the coefficient of $i$ to $C_{z} ; \alpha, \beta$ are the angle of attack and sideslip angle.

In order to build a model of the UAV attitude system, define $\quad x_{1}=[\vartheta, \varphi, \gamma]^{T}, x_{2}=\left[\omega_{x}, \omega_{y}, \omega_{z}\right]^{T}, \quad$ and $u=$ $\left[\begin{array}{llllll}\delta_{a 1} & \delta_{a 1} & \delta_{a 2} & \delta_{b 1} & \delta_{b 2} & \delta_{y}\end{array}\right]^{T}$. Then, the UAV attitude dynamic equation with time-varying interference can be expressed as

$$
\left\{\begin{array}{l}
\dot{x}_{1}=A(t) x_{2}+d_{1}(t) \\
\dot{x}_{2}=B(t) u(t)+f\left(x_{1}, x_{2}\right)+\Delta f\left(x_{1}, x_{2}\right)+d_{2}(t) \\
y=x_{1}
\end{array}\right.
$$

where $A(t) \in \mathbb{R}^{3 \times 3}, d_{1}(t) \in \mathbb{R}^{3 \times 1}, B(t) \in \mathbb{R}^{3 \times 5}$, and $f(x) \in$ $\mathbb{R}^{3 \times 1}$. They are denoted by 


$$
\begin{aligned}
& A(t)=\left[\begin{array}{ccc}
0 & \sin \gamma & \cos \gamma \\
0 & \frac{\cos \gamma}{\cos \vartheta} & -\frac{\sin \gamma}{\cos \vartheta} \\
1 & -\tan \vartheta \cos \gamma & \tan \vartheta \sin \gamma
\end{array}\right], \\
& B(t)=\bar{q} S b I^{-1}\left[\begin{array}{ccccc}
C_{x}^{\delta_{a 1}} & C_{x}^{\delta_{a 2}} & C_{x}^{\delta_{b 1}} & C_{x}^{\delta_{b 2}} & 0 \\
0 & 0 & 0 & 0 & C_{y}^{\delta_{y}} \\
C_{z}^{\delta_{a 1}} & C_{z}^{\delta_{a 2}} & C_{z}^{\delta_{b 1}} & C_{z}^{\delta_{b 2}} & 0
\end{array}\right] \\
& f\left(x_{1}, x_{2}\right)=I^{-1}\left\{\bar{q} S b\left[\begin{array}{c}
C_{x}^{\beta} \beta \\
C_{y}^{\beta} \beta \\
C_{z 0}+C_{z}^{\alpha} \alpha
\end{array}\right]+\Omega_{I} \times\left(I * \Omega_{I}\right)\right\} \\
& \Delta f\left(x_{1}, x_{2}\right)=\bar{q} S b I^{-1}\left[\begin{array}{c}
\Delta_{x} \\
\Delta_{y} \\
\Delta_{z}
\end{array}\right] .
\end{aligned}
$$

All states are bound to the compact set

$$
\Omega_{x}:=\left\{x_{i} \in R,\left|x_{i}(t)\right| \leq b_{c i}, i=1,2\right\},
$$

where $b_{c i}$ is a known positive constant.

Remark 1. In fact, it is difficult to develop an exact model for the UAV since we cannot reproduce the complex flight environment of the UAV in a wind tunnel. So, the unmodeled dynamics $d_{i}$ and uncertain parameters $\Delta_{i}$ are inevitable.

2.2. Assumptions and Lemmas. The purpose of this paper is to design a controller $u$ : (1) for any initial state, there is a finite time $T>0$ so that the output tracking error meets $\left|y(t)-y_{d}(t)\right| \leq \varepsilon, \forall t \geq T$, where $y_{d}(t)$ is the desired reference trajectory; (2) all closed-loop signals are bounded; (3) state constraint requirements are not violated.

In order to design the controller, analyze the stability of the system, and achieve the above control objectives, the following assumption and lemmas are introduced.

Assumption 1. For a continuously differentiable expected trajectory $y_{d}(t)$, there exists known positive $y_{0}$ such that $\left|y_{d}(t)\right| \leq y_{0},\left|\dot{y}_{d}(t)\right| \leq y_{0}$, and $y_{0}<b_{c_{i}}$.

Lemma 1. According to Young's inequality, given any $a, b \in \mathbb{R}\{\hat{3} \times 3\}, p, q>1$ and $1 / p+1 / q=1$,

$$
a b \leq \varepsilon a^{p}+c_{\varepsilon} b^{q},
$$

where $\varepsilon$ is arbitrarily small and $c_{\varepsilon}$ is arbitrarily large, and $c_{\varepsilon}=p \varepsilon^{-(q / p q)}$.
Lemma 2. According to Young's inequality, for given positive integers $m, n$ and for each $x \in \mathbb{R}, y \in \mathbb{R}$, the following inequality holds:

$$
|x|^{m}|y|^{n} \leq \frac{1}{\gamma_{1}} \frac{m}{m+n} \gamma_{2}^{-(n / m)}|x|^{m+n}+\gamma_{2} \frac{n}{m+n} \gamma_{1}^{m / n}|y|^{m+n},
$$

where $\gamma_{1}>0$ and $\gamma_{2}>0$ can be any constant or real-valued function.

Lemma 3. For any given constant $\varepsilon>0$ and $z \in \mathbb{R}$, there is

$$
0 \leq|z|-z \tanh \left(\frac{z}{\varepsilon}\right) \leq \kappa \varepsilon,
$$

where $\kappa$ is a constant that satisfies $\kappa=e^{-(\kappa+1)}$; for instance, $\kappa=0.2785$.

2.3. Fuzzy Logic Theory. The specific form of the fuzzy logic system is as follows, which is composed of single point fuzzy set, product inference rule, center average weighted nonfuzzy set and Gaussian membership function:

$$
y(x)=\frac{\sum_{i=1}^{N} \Phi_{i} \prod_{j=1}^{n} \mu_{F_{j}^{i}}\left(x_{j}\right)}{\sum_{i=1}^{N}\left[\prod_{j=1}^{n} \mu_{F_{j}^{i}}\left(x_{j}\right)\right]} .
$$

Let

$$
\psi_{i}(x)=\frac{\prod_{j=1}^{n} \mu_{F_{j}^{i}}\left(x_{j}\right)}{\sum_{i=1}^{N}\left[\prod_{j=1}^{n} \mu_{F_{j}^{i}}\left(x_{j}\right)\right]} .
$$

$\Psi(x)=\left[\psi_{1}(x), \psi_{2}(x), \ldots, \psi_{N}(x)\right]^{T}$

and $\Phi=\left[\Phi_{1}, \ldots, \Phi_{N}\right]^{T}$. Then, the fuzzy system is

$$
y(x)=\Phi^{T} \Psi(x) .
$$

Lemma 4. There is a fuzzy system $\Phi^{T} \Psi(x)$; for any given constant $\varepsilon>0$, there is

$$
\sup _{x \in \Omega}\left|f(x)-\Phi^{T} \Psi(x)\right| \leq \varepsilon,
$$

where $f(x)$ is a continuous function defined on the compact set $\Omega$.

\section{Main Results}

Next, introduce the following transformations:

$$
\left\{\begin{array}{l}
e_{1}=x_{1}-y_{d} \\
e_{2}=x_{2}-x_{2 c}
\end{array}\right.
$$

where $e_{1}, e_{2} \in \mathbb{R}^{3 \times 1}$ and $x_{2 c} \in \mathbb{R}^{3 \times 1}$, respectively, represent virtual tracking errors and virtual control signal. Furthermore, $\left|x_{2 c}\right|<x_{20}{ }^{*}$ is constructed, where $x_{20}{ }^{*}>0$ is a constant. The following high-order tan-type BLFs are introduced to solve the problem of system state constraints of the fixed-wing UAV system: 


$$
V_{i j}^{*}=\frac{2 b_{i}^{5-p}}{a \pi\left(5-p_{i}\right)} \tan \left(\frac{\pi e_{i j}^{5-p_{i}}}{2 b_{i}^{5-p_{i}}}\right)+\frac{\widetilde{D}_{i j}^{2}}{2 \eta_{i j}} .
$$

For each $k=1,2, \ldots, n$, define $V_{k=} \sum_{i=1}^{k} V_{i}^{*}$, where $p_{i} \in R_{\text {odd }}^{\geq 1}:=(p / q) \mid p, q$ are odd positive integers and $p \geq q, b_{i}=b_{c_{i}}-\alpha_{i_{0}}^{*}, i$ is the order of the system, $j$ is the dimension of the system, and $\alpha_{i 0}^{*}>0$ is a constant that will be given later. For the second-order and three-dimensional UAV system in this article, $p_{1}=1$ and $p_{2}=3$.

Remark 2. Tan-type BLFs (15) are proposed to handle fullstate constraints for high-order systems, where $b_{i}=b_{c_{i}}-\alpha_{i_{0}}^{*}$. Because $\quad b_{c i} \longrightarrow \infty$ means $b_{i} \longrightarrow \infty, \tan \left(\left[\left(\pi e_{i j}^{5-p_{i}}\right) /\right.\right.$ $\left.\left.\left(2 b_{i}^{5-p_{i}}\right)\right]\right) \sim\left[\left(\pi e_{i j}^{5-p_{i}}\right) /\left(2 b_{i}^{5-p_{i}}\right)\right]$ when $b_{i} \longrightarrow \infty$. From this, the formula can be further inferred as follows:

$$
\lim _{b_{i}} \frac{2 b_{i}^{5-p_{i}}}{a \pi\left(5-p_{i}\right)} \tan \left(\frac{\pi e_{i}^{5-p_{i}}}{2 b_{i}^{5-p_{i}}}\right)=\frac{e_{i}^{5-p_{i}}}{a\left(5-p_{i}\right)} .
$$

Therefore, the high-order tan BLF introduced in this paper will be transformed into a general Lyapunov function, and the design method is also effective for unconstrained high-order systems.

The parameters used in the subsequent control design are defined as follows:

$$
\varphi_{i j}=\cos ^{2}\left(\left[\frac{\left(\pi e_{i j}^{5-p_{i}}\right)}{\left(2 b_{i}^{5-p_{i}}\right)}\right]\right), \quad i=1,2 ., l_{1 j}=\frac{\varepsilon}{a \varphi_{1 j}},
$$

where $a$ and $\varepsilon$ are adjustment parameters.

$$
\begin{aligned}
\dot{V}_{1 j} & =\frac{e_{1 j}^{3}}{a \varphi_{1 j}}\left(x_{2 j}+d_{1 j}(t)-\dot{y}_{j d}\right)+\frac{\widetilde{D}_{1 j} \dot{\hat{D}}_{1 j}}{\eta_{1 j}} \\
& =-k_{1 j} \tan \left(\frac{\pi e_{1 j}^{4}}{2 b_{1}^{4}}\right)-l_{1 j} e_{1 j}^{4}+\frac{e_{1 j}^{3}}{a \varphi_{1 j}}\left(x_{2 j}+d_{1 j}(t)+\bar{f}_{1 j}\right)+\frac{\widetilde{D}_{1 j} \dot{\hat{D}}_{1 j}}{\eta_{1 j}}
\end{aligned}
$$

where $\bar{f}_{1 j}=-\dot{y}_{j d}-a \varphi_{1 j} l_{1 j} e_{1 j}+a k_{1} \sin (\tau) \cos (\tau) / e_{1 j}^{3}, j=$ $1,2,3, \tau=\left(\pi e_{1 j}^{4} / 2 b_{1}^{4}\right)$.

Using the fuzzy system $\Phi_{1 j}^{T} \Psi_{1 j}\left(X_{1 j}\right)$, it is easy to get

$$
\bar{f}_{1 j}=\Phi_{1 j}^{T} \Psi_{1 j}\left(X_{1 j}\right)+\tau_{1 j}\left(X_{1 j}\right),
$$

where $\left|\tau_{1 j}\left(X_{1 j}\right)\right| \leq \varepsilon_{1 j}$ are the approximation errors. This comes together with Lemma 2:

$$
\begin{aligned}
\frac{e_{1 j}^{3}}{a \varphi_{1 j}}\left(\Phi_{1 j}^{T} \Psi_{1 j}\left(X_{1 j}\right)+\tau_{1 j}\left(X_{1 j}\right)\right) \leq & \frac{\left|e_{1 j}\right|^{3}}{a \varphi_{1 j}}\left(\left\|\Phi_{1 j}\right\|\left\|\Psi_{1 j}\right\|+\varepsilon_{1 j}\right) \leq \frac{1}{\Gamma} \frac{1}{4} \varsigma_{1 j}^{-3}\left(\frac{\left\|\Phi_{1 j}\right\|}{a}\right)^{4} \\
& +\varsigma_{1 j} \frac{3}{4} \Gamma^{1 / 3}\left(\frac{\left\|\Psi_{1 j}\right\|}{\varphi_{1 j}}\right)^{4 / 3} e_{1 j}^{4}+\frac{1}{\Gamma} \frac{1}{4} \varsigma_{1 j}^{-3}\left(\frac{\varepsilon_{1 j}}{a}\right)^{4}+\varsigma_{1 j} \frac{3}{4} \Gamma^{1 / 3}\left(\frac{1}{\varphi_{1 j}}\right)^{4 / 3} e_{1 j}^{4} \\
\leq & \frac{\xi_{1 j}}{\Gamma}+\rho_{1 j}(\cdot) e_{1 j}^{4}
\end{aligned}
$$

where $\Gamma>0$ and $\varsigma_{1 j}>0$ are arbitrary constants. $\xi_{1 j}=4 \zeta_{1 j}^{-3}$ $\left(\left(\left\|\Phi_{1 j}\right\| / a\right)^{4}+\left(\varepsilon_{1 j} / a\right)^{4}\right), \rho_{1 j}(\cdot)=\varsigma_{1 j} 3 / 4 \Gamma^{1 / 3}\left(\left(\left\|\Psi_{1 j}\right\| / \varphi_{1 j}\right)^{4 / 3}+\right.$ $\left.\left(1 / \varphi_{1 j}\right)^{4 / 3}\right)$.

Substitute (20) into (18) to get

$$
\dot{V}_{1 j} \leq-k_{1 j} \tan \left(\frac{\pi e_{1 j}^{4}}{2 b_{1}^{4}}\right)+\frac{e_{1 j}^{4}}{a \varphi_{1 j}}\left(e_{2 j}+d_{1 j}(t)+x_{2 j c}\right)+\rho_{1 j}(\cdot) e_{1 j}^{4}+\frac{\xi_{1 j}}{\Gamma}-l_{1 j} e_{1 j}^{4}+\frac{\widetilde{D}_{1 j} \dot{\hat{D}}_{1 j}}{\eta_{1 j}}
$$


where $e_{2 j}=x_{2 j}-x_{2 j c}$. Define

$$
D_{1 j}=\sup _{t \geq 0}\left\|d_{1 j}(t)\right\| \text {. }
$$

$$
\frac{e_{1}^{p-p_{1}+1}}{a \varphi_{1}} d_{1}(t) \leq\left\|\frac{e_{1}^{p-p_{1}+1}}{a \varphi_{1}}\right\|\left\|d_{1}(t)\right\| \leq\left\|\frac{e_{1 j}^{3}}{a \varphi_{1 j}}\right\| D_{1 j} \leq D_{1 j} \frac{e_{1 j}^{3}}{a \varphi_{1 j}} \tanh \left(\frac{e_{1 j}^{3}}{a \varphi_{1 j} \varepsilon_{1 j}}\right)+\kappa D_{1 j} \varepsilon_{1 j} .
$$

$x_{2 j c}$ is designed as

In this way,

$$
x_{2 j c}=-a \varphi_{1 j} \rho_{1 j}(\cdot) e_{1 j}-\widehat{D}_{1 j} \tanh \left(\frac{e_{1 j}^{3}}{a \varphi_{1 j} \varepsilon_{1 j}}\right) .
$$

$$
\begin{aligned}
\frac{e_{1 j}^{3}}{a \varphi_{1 j}}\left(d_{1 j}(t)+x_{2 j c}\right) \leq & D_{1 j} \frac{e_{1 j}^{3}}{a \varphi_{1 j}} \tanh \left(\frac{e_{1 j}^{3}}{a \varphi_{1 j} \varepsilon_{1 j}}\right)+\kappa D_{1 j} \varepsilon_{1 j}-\frac{e_{1 j}^{3}}{a \varphi_{1 j}} a \varphi_{1 j} \rho_{1 j}(\cdot) e_{1 j} \\
& -\frac{e_{1 j}^{3}}{a \varphi_{1 j}} \widehat{D}_{1 j} \tanh \left(\frac{e_{1 j}^{3}}{a \varphi_{1 j} \varepsilon_{1 j}}\right) \leq-e_{1 j}^{4} \rho_{1 j}(\cdot)-\widetilde{D}_{1 j} \frac{e_{1 j}^{3}}{a \varphi_{1 j}} \tanh \left(\frac{e_{1 j}^{3}}{a \varphi_{1 j} \varepsilon_{1 j}}\right)+\kappa D_{1 j} \varepsilon_{1 j} .
\end{aligned}
$$

Substituting (25) into (21), we have

$$
\begin{aligned}
\dot{V}_{1 j} \leq & -k_{1 j} \tan \left(\frac{\pi e_{1 j}^{4}}{2 b_{1}^{4}}\right)+\frac{e_{1 j}^{3}}{a \varphi_{1 j}} e_{2 j}+\frac{\xi_{1 j}}{\Gamma}-l_{1 j} e_{1 j}^{4}-\widetilde{D}_{1 j} \frac{e_{1 j}^{3}}{a \varphi_{1 j}} \tanh \left(\frac{e_{1 j}^{3}}{a \varphi_{1 j} \varepsilon_{1 j}}\right) \\
& +\kappa D_{1 j} \varepsilon_{1 j}+\frac{\widetilde{D}_{1 j} \dot{\hat{D}}_{1 j}}{\eta_{1 j}} .
\end{aligned}
$$

The adaptive law is as follows:

So, it can be deduced that

$$
\dot{\hat{D}}_{1 j}=\eta_{1 j}\left(\frac{e_{1 j}^{3}}{a \varphi_{1 j}} \tanh \left(\frac{e_{1 j}^{3}}{a \varphi_{1 j} \varepsilon_{1 j}}\right)-\sigma_{1 j} \widehat{D}_{1 j}\right) .
$$

$$
\dot{V}_{1 j} \leq-k_{1 j} \tan \left(\frac{\pi e_{1 j}^{4}}{2 b_{1}^{4}}\right)+\frac{e_{1 j}^{3}}{a \varphi_{1 j}} e_{2 j}+\frac{\xi_{1 j}}{\Gamma}-l_{1 j} e_{1 j}^{4}-\sigma_{1 j} \widetilde{D}_{1 j} \widehat{D}_{1 j}+\kappa D_{1 j} \varepsilon_{1 j} .
$$

as

Given $-\widetilde{D}_{1 j} \widehat{D}_{1 j} \leq-\widetilde{D}_{1 j}^{2} / 2+D_{1 j}^{2} / 2,(28)$ can be rewritten

$$
\dot{V}_{1 j} \leq-k_{1 j} \tan \left(\frac{\pi e_{1 j}^{4}}{2 b_{1}^{4}}\right)+\frac{e_{1 j}^{3}}{a \varphi_{1 j}} e_{2 j}-l_{1 j} e_{1 j}^{4}-\frac{\sigma_{1 j} \widetilde{D}_{1 j}^{2}}{2}+\frac{\xi_{1 j}}{\Gamma}+\frac{\sigma_{1 j} D_{1 j}^{2}}{2}+\bar{\xi}_{1 j}
$$


where $\bar{\xi}_{1 j}=\kappa D_{1 j} \varepsilon_{1 j}$. Then, the asymptotic bounded convergence of the system is proved.

Remark 3. The negative term $-l_{1 j} e_{1 j}^{4}$ designed in (29) supplies an adequate stable domain to stabilize the positive terms containing $e_{1 j}$ which will appear in the next design step.

Next, consider the design of $u$.

$$
\dot{V}_{2 j}=\dot{V}_{1 j}+\dot{V}_{2 j}^{*}=\dot{V}_{1 j}+\frac{e_{2 j}}{a \varphi_{2 j}}\left(u_{j}+f(x)+d_{2}(t)-\dot{x}_{2 j c}\right)+\frac{\widetilde{D}_{2 j} \dot{\hat{D}}_{2 j}}{\eta_{2 j}}
$$

By substituting (29) into (30), it can be obtained that

$$
\begin{aligned}
\dot{V}_{2 j} \leq & -k_{1 j} \tan \left(\frac{\pi e_{1 j}^{4}}{2 b 4}\right)+\frac{e_{1 j}^{3}}{a \varphi_{1 j}} e_{2 j}+\frac{e_{2 j}}{a \varphi_{2 j}}\left(u_{j}+f(x)+d_{2}(t)-\dot{x}_{2 j c}\right) \\
& +\frac{\xi_{1 j}}{\Gamma}-l_{1 j} e_{1 j}^{4}+\bar{\xi}_{1 j}+\frac{\widetilde{D}_{2 j} \dot{\hat{D}}_{2 j}}{\eta_{2 j}} .
\end{aligned}
$$

In accordance with Lemma 1,

$\frac{e_{1 j}^{3}}{a \varphi_{1 j}} e_{2 j} \leq \frac{1}{a \varphi_{1 j}}\left(\varepsilon\left(e_{1 j}^{3}\right)^{m}+C_{\varepsilon}\left(e_{2 j}\right)^{n}\right) \leq \rho_{21 j}(\cdot) e_{2 j}^{n}+l_{1 j} e_{1 j}^{3 m}$, where $m=4 / 3, n=4, C_{\varepsilon}=(n \varepsilon)^{-n / m n}, \rho_{21 j}(\cdot)=C_{\varepsilon} \quad \mid a \varphi_{1 j}$, and $l_{1 j}=\varepsilon / a \varphi_{1 j}$.

Substituting (32) into (31),

$$
\dot{V}_{2 j} \leq-k_{1 j} \tan \left(\frac{\pi e_{1 j}^{4}}{2 b_{1}^{4}}\right)+\rho_{21 j}(\cdot) e_{2 j}^{n}+\frac{\xi_{1 j}}{\Gamma}+\bar{\xi}_{1 j}+\frac{e_{2 j}}{a \varphi_{2 j}}\left(u_{j}+f(x)+d_{2 j}(t)-\dot{x}_{2 j c}\right)+\frac{\widetilde{D}_{2 j} \dot{\hat{D}}_{2 j}}{\eta_{2 j}} .
$$

Further deformation of the above equation can be obtained:

$$
\begin{aligned}
\dot{V}_{2 j} \leq & -k_{1 j} \tan \left(\frac{\pi e^{4}}{2 b_{1}^{4}}\right)-k_{2 j} \tan \left(\frac{\pi e_{2 j}^{2}}{2 b_{2}^{2}}\right)+\rho_{21 j}(\cdot) e_{2 j}^{n}+\frac{\xi_{1 j}}{\Gamma}+\bar{\xi}_{1 j}-\frac{\sigma_{1 j} \widetilde{D}_{1 j}^{2}}{2}+\frac{\sigma_{1 j} D_{1 j}^{2}}{2} \\
& -\frac{\sigma_{1 j} \widetilde{D}_{1 j}^{2}}{2}+\frac{\sigma_{1 j} D_{1 j}^{2}}{2}+\frac{e_{2 j}}{a \varphi_{2 j}}\left(u_{j}+f(x)+d_{2 j}(t)\right)+\frac{e_{2 j}}{a \varphi_{2 j}}\left(\bar{f}_{2 j}\right)+\frac{\widetilde{D}_{2 j} \dot{\hat{D}}_{2 j}}{\eta_{2 j}},
\end{aligned}
$$


where $\bar{f}_{2 j}=-\dot{x}_{2 j c}+a k_{2 j} \sin (\tau) \cos (\tau) / e_{2 j}$.

Use the fuzzy system $\Phi_{2 j}^{T} \Psi_{2 j}\left(X_{2 j}\right)$ to get

$$
\bar{f}_{2 j}=\Phi_{2 j}^{T} \Psi_{2 j}\left(X_{2 j}\right)+\tau_{2 j}\left(X_{2 j}\right)
$$

where $\left|\tau_{2 j}\left(X_{2 j}\right)\right| \leq \varepsilon_{2 j}$ is the approximation error; through Lemma 2, we get

$$
\begin{aligned}
\frac{e_{2 j}}{a \varphi_{2 j}}\left(\Phi_{2 j}^{T} \Psi_{2 j}+\tau_{2 j}\right) \leq & \frac{\left|e_{2 j}\right|}{a \varphi_{2 j}}\left(\left\|\Phi_{2 j}\right\|\left\|\Psi_{2 j}\right\|+\varepsilon_{2 j}\right) \leq \frac{1}{\Gamma} \frac{3}{4} \zeta_{22}^{-(1 / 3)}\left(\frac{\left\|\Phi_{2 j}\right\|}{a}\right)^{4 / 3} \\
& +\varsigma_{22} \frac{1}{4} \Gamma^{3}\left(\frac{\left\|\Psi_{2 j}\right\|}{\varphi_{2 j}}\right)^{4} e_{2 j}^{4}+\frac{1}{\Gamma} \frac{3}{4} \zeta_{2 j}^{-(1 / 3)}\left(\frac{\varepsilon_{2 j}}{a}\right)^{4 / 3} \\
& +\varsigma_{2 j} \frac{1}{4} \Gamma^{3}\left(\frac{1}{\varphi_{2 j}}\right)^{4} e_{2 j}^{4} \\
= & \frac{\xi_{2 j}}{\Gamma}+\rho_{2 j}(\cdot) e_{2 j}^{4},
\end{aligned}
$$

where $\quad \xi_{2 j}=3 / 4 \zeta_{2 j}^{-(1 / 3)} \quad\left(\left(\left\|\Phi_{2 j}\right\| / a\right)^{4 / 3}+\left(\varepsilon_{2 j} / a\right)^{4 / 3}\right), \rho_{2 j}=$ $\varsigma_{2 j} 1 / 4 \Gamma^{3}\left(\left(\left\|\Psi_{2 j}\right\| / \varphi_{2 j}\right)^{4}+\left(1 / \varphi_{2 j}\right)^{4}\right), \Gamma>0$, and $\varsigma_{2 j}>0$, being adjustment parameters. Substituting (36) into (34) results in

$$
\begin{aligned}
\dot{V}_{2 j} \leq & -k_{1 j} \tan \left(\frac{\pi e_{1 j}^{4}}{2 b_{1}^{4}}\right)-k_{2 j} \tan \left(\frac{\pi e_{2 j}^{2}}{2 b_{2}^{2}}\right)+\rho_{21 j}(\cdot) e_{2 j}^{n}+\rho_{2 j}(\cdot) e_{2 j}^{4}+\frac{\xi_{j}}{\Gamma}+\bar{\xi}_{1 j} \\
& +\frac{e_{2 j}}{a \varphi_{2 j}}\left(m_{j}+d_{2 j}(t)\right)+\frac{\widetilde{D}_{2 j} \dot{\hat{D}}_{2 j}}{\eta_{2 j}}-\frac{\sigma_{1 j} \widetilde{D}_{1 j}^{2}}{2}+\frac{\sigma_{1 j} D_{1 j}^{2}}{2},
\end{aligned}
$$

where $\xi_{j}=\xi_{1 j}+\xi_{2 j}$.

Same as above, according to Lemma 3, define $D_{2 j}=$ $\sup _{t \geq 0}\left\|d_{2 j}(t)\right\|$ so that

$$
\frac{e_{2 j}}{a \varphi_{2 j}} d_{2 j}(t) \leq\left\|\frac{e_{2 j}}{a \varphi_{2 j}}\right\|\left\|d_{2 j}(t)\right\| \leq\left\|\frac{e_{2 j}}{a \varphi_{2 j}}\right\| D_{2 j} \leq D_{2 j} \frac{e_{2 j}}{a \varphi_{2 j}} \tanh \left(\frac{e_{2 j}}{a \varphi_{2 j} \varepsilon_{2 j}}\right)+\kappa D_{2 j} \varepsilon_{2 j} .
$$


Design $u_{j}$ as

Then,

$$
u_{j}=-a \varphi_{2 j} e_{2 j}^{3}\left(\rho_{21 j}+\rho_{2 j}\right)-\widehat{D}_{2 j} \tanh \left(\frac{e_{2 j}}{a \varphi_{2 j} \varepsilon_{2 j}}\right) \text {. }
$$

$$
\begin{aligned}
\frac{e_{2 j}}{a \varphi_{2 j}}\left(d_{2 j}(t)+m_{j}\right) \leq & D_{2 j} \frac{e_{2 j}}{a \varphi_{2 j}} \tanh \left(\frac{e_{2 j}}{a \varphi_{2 j} \varepsilon_{2 j}}\right)+\kappa D_{2 j} \varepsilon_{2 j}-\frac{e_{2 j}}{a \varphi_{2 j}} a \varphi_{2 j} e_{2 j}^{3}\left(\rho_{21 j}+\rho_{2 j}\right) \\
& -\frac{e_{2 j}}{a \varphi_{2 j}} \widehat{D}_{2 j} \tanh \left(\frac{e_{2}}{a \varphi_{2 j} \varepsilon_{2 j}}\right) \\
\leq & -e_{2 j}^{4}\left(\rho_{21 j}+\rho_{2 j}\right)-\widetilde{D}_{2 j} \frac{e_{2}}{a \varphi_{2 j}} \tanh \left(\frac{e_{2 j}}{a \varphi_{2 j} \varepsilon_{2 j}}\right)+\kappa D_{2 j} \varepsilon_{2 j} .
\end{aligned}
$$

This together with (37) yields

$$
\begin{aligned}
\dot{V}_{2 j} \leq & -k_{1 j} \tan \left(\frac{\pi e_{1 j}^{4}}{2 b_{1}^{4}}\right)-k_{2 j} \tan \left(\frac{\pi e_{2 j}^{2}}{2 b_{2}^{2}}\right)+\frac{\xi_{j}}{\Gamma}+\bar{\xi}_{1 j}-\widetilde{D}_{2 j} \frac{e_{2 j}}{a \varphi_{2 j}} \tanh \left(\frac{e_{2 j}}{a \varphi_{2 j} \varepsilon_{2 j}}\right) \\
& +\kappa D_{2 j} \varepsilon_{2 j}+\frac{\widetilde{D}_{2 j} \dot{\widehat{D}}_{2 j}}{\eta_{2 j}}-\frac{\sigma_{1 j} \widetilde{D}_{1 j}^{2}}{2}+\frac{\sigma_{1 j} D_{1 j}^{2}}{2} .
\end{aligned}
$$

Design the adaptive law as

$$
\dot{\hat{D}}_{2 j}=\eta_{2 j}\left(\frac{e_{2 j}}{a \varphi_{2 j}} \tanh \left(\frac{e_{2 j}}{a \varphi_{2 j} \varepsilon_{2 j}}\right)-\sigma_{2 j} \widehat{D}_{2 j}\right) \text {. }
$$

So, it can be deduced that

$$
\begin{aligned}
\dot{V}_{2 j} \leq & -k_{1 j} \tan \left(\frac{\pi e_{1}^{4}}{2 b_{1}^{4}}\right)-k_{2 j} \tan \left(\frac{\pi e_{2 j}^{2}}{2 b_{2}^{2}}\right)+\frac{\xi_{j}}{\Gamma}+\bar{\xi}_{1 j}+\kappa D_{2 j} \varepsilon_{2 j} \\
& -\sigma_{2 j} \widetilde{D}_{2 j} \widehat{D}_{2 j}-\frac{\sigma_{1 j} \widetilde{D}_{1 j}^{2}}{2}+\frac{\sigma_{1 j} D_{1 j}^{2}}{2} .
\end{aligned}
$$

In the same way, $-\widetilde{D}_{2 j} \widehat{D}_{2 j} \leq-\widetilde{D}_{2 j}^{2} / 2+D_{2 j}^{2} / 2$. Therefore, (43) can be rewritten as

$$
\begin{aligned}
\dot{V}_{2 j} \leq & -k_{1 j} \tan \left(\frac{\pi e_{1 j}^{4}}{2 b_{1}^{4}}\right)-k_{2 j} \tan \left(\frac{\pi e_{2 j}^{2}}{2 b_{2}^{2}}\right)+\frac{\xi_{j}}{\Gamma}+\bar{\xi}_{1 j}+\bar{\xi}_{2 j} \\
& -\frac{\sigma_{2 j} \widetilde{D}_{2 j}^{2}}{2}+\frac{\sigma_{2 j} D_{2 j}^{2}}{2}-\frac{\sigma_{1 j} \widetilde{D}_{1 j}^{2}}{2}+\frac{\sigma_{1 j} D_{1 j}^{2}}{2} .
\end{aligned}
$$

Then, the asymptotic bounded convergence of the system can be proved.

Because the system is a second-order three-dimensional system, $V_{1 j}, V_{2 j}$ are asymptotically bounded convergences, and $V_{1}=V_{11}+V_{12}+V_{13}, \quad V_{2}^{*}=V_{21}+V_{22}+V_{23}$. So, the system is also asymptotically bounded. According to $V_{k=} \sum_{i=1}^{k} V_{i}^{*}$, we know that $V_{2}=V_{1}+V_{2}^{*}$. Then,

$$
\begin{aligned}
& \dot{V}_{2} \leq-\sum_{j=1}^{3}\left(\begin{array}{c}
k_{1 j} \tan \left(\frac{\pi e_{1 j}^{4}}{2 b_{1}^{4}}\right)+k_{2 j} \tan \left(\frac{\pi e_{2 j}^{2}}{2 b_{2}^{2}}\right)+\frac{\sigma_{2 j} \widetilde{D}_{2 j}^{2}}{2}-\frac{\sigma_{2 j} D_{2 j}^{2}}{2}- \\
\frac{\sigma_{1 j} \widetilde{D}_{1 j}^{2}}{2}+\frac{\sigma_{1 j} D_{1 j}^{2}}{2}+\bar{\xi}_{1 j}+\bar{\xi}_{2 j}+\frac{\xi_{j}}{\Gamma}
\end{array}\right) \\
& \leq-c V_{2}+\frac{\xi_{n}}{\Gamma}+\bar{\xi}_{n},
\end{aligned}
$$


where $\xi_{n}=\sum_{j=1}^{3} \xi_{j}, \bar{\xi}_{n}=\sum_{j=1}^{3} \bar{\xi}_{i j}, i=1,2$, and $\quad c=\min _{\substack{1 \leq i \leq 2 \\ 1 \leq j \leq 3}}$ $\left\{a k_{i j} \pi\left(p-p_{i}+2\right) / 2 b_{i}^{p-p_{i}+2}\right\}$.

Proof. If the system with full-state constraints meets the assumptions, the trace controller $u$ can accomplish the following:

(i) All states of a closed-loop system are bounded

(ii) It will not violate the complete state constraints

(iii) The tracking errors $e_{1}, e_{2}$ converge to any small neighborhood of zero

(iv) The system is stable under time-varying disturbance

Considering $\Gamma \geq 1$, according to formula (45), we can get $\dot{V}_{2} \leq-c V_{2}+\xi_{n}+\bar{\xi}_{n}$. Therefore, we can infer that $V_{2} \leq\left(V_{2}(0)-\left(\left(\xi_{n}+\bar{\xi}_{n}\right) / c\right) e^{-c t}+\left(\left(\xi_{n}+\bar{\xi}_{n}\right) / c\right)\right.$. Hence, $V_{2}$ is constrained; it also represents that $\tan \left(\left(\pi e_{i}^{p-p_{i}+2}\right) /\left(2 b_{i}^{p-p_{i}+2}\right)\right)$ is bounded. Therefore, $\left|e_{i}\right|<b_{i}$. According to the Assumption 1, we can know that $\left|x_{1}\right| \leq\left|e_{1}\right|+\left|y_{d}\right|<b_{1}+y_{0}=b_{c_{1}}$. On the contrary, it can be inferred from the continuity of $x_{2 c}$ and the boundedness of $x_{1}, y_{d}, \widehat{D_{1 j}}$ that $x_{2 c}$ is also constrained. Hence, there is a finite constant $x_{2 c 0}^{*}$ that makes $\left|x_{2 c}\right| \leq x_{2 c 0}^{*}$. From the boundedness and error transformation of $e_{2}$ and $x_{2 c}$, the conclusion of $\left|x_{2}\right| \leq\left|e_{2}\right|+\left|x_{2 c}\right|<b_{2}+x_{2 c 0}=b_{c_{2}}$ can be drawn. As a result, all states are bounded and meet the constraints.

Remark 4. For the possible disturbance $d_{i}(t)$, it is replaced by sup $\left\|d_{i}(t)\right\|$, which greatly reduces the computation. If the adatefive parameters are used to cancel the disturbance, the adaptive parameters should be designed for $d_{i}(t)$, respectively, so that the computation burden will be increased.

\section{Simulation Experiment}

4.1. Initial Value Selection. In order to verify the effectiveness and superiority of the HOBLF method proposed in this paper under the influence of time-varying interference, this section studies the simulation effect under the influence of strong time-varying interference and weak time-varying interference, respectively, to verify its validity. Under the same conditions, the simulation effects of the proposed method and DOBC, general adaptive control, and low-order general BLF were compared to verify its superiority.

Now, suppose that the UAV rises at a fixed attitude angle in the air, and the UAV needs to change from the rising stage to the flat flying stage. The square wave signal filtered by the second-order link is selected as the expected signal with an amplitude of 1 and a period of $20 \mathrm{~s}$. Related parameters have been listed in Tables 1 and 2 .

4.2. Simulation Comparison. The effectiveness of the HOBLF is verified by performing the simulation under three cases.

Case 1. First, consider the case of using the HOBLF for control under weak time-varying interference. For pitch angle $\vartheta$, yaw angle $\psi$, and roll angle $\gamma$, the time-varying interferences are $0.01 \sin (t), 0.02 \sin (t)$, and $0.03 \sin (t)$, respectively.
Case 2. Then, consider the case of controlling with the HOBLF under strong time-varying interference. For pitch angle $\vartheta$, yaw angle $\psi$, and roll angle $\gamma$, the time-varying interferences are $0.1 \sin (t), 0.2 \sin (t)$, and $0.3 \sin (t)$, respectively.

The simulation results are shown in Figures 2-5. It can be analyzed from Figures 2-5 that, under the influence of strong or weak time-varying interference, the controlled states $\vartheta, \psi$, and $\gamma$ can track the expected signal $\vartheta_{d}, \psi_{d}$, and $\gamma_{d}$ smoothly without violating the state constraint. Therefore, the effectiveness and superiority of the HOBLF can be verified.

Case 3. high-order tan-type BLF is selected. The tangent Lyapunov function can not only deal with the adaptive control of nonlinear systems with state constraints but also can be regarded as a quadratic Lyapunov function without constraints. Therefore, the tangential Lyapunov function can deal with constrained and unconstrained systems. The state constraint $b_{c 1}$ on $x_{1}$ is 1.5 .

The design of inside loop controller $x_{2 c}$ is as follows:

$$
\begin{aligned}
\varphi_{1} & =\cos ^{2}\left(\frac{\pi e_{1}^{4}}{2 b_{1}^{4}}\right), \\
\rho_{1 j}(\cdot) & =\varsigma_{11} \frac{3}{4} \Gamma^{1 / 3}\left(\left(\frac{\left\|\Psi_{1 j}\right\|}{\varphi_{1 j}}\right)^{4 / 3}+\left(\frac{1}{\varphi_{1 j}}\right)^{4 / 3}\right), \\
n_{j c} & =-a \varphi_{1 j} \rho_{1 j}(\cdot) e_{1 j}-\widehat{D}_{1 j} \tanh \left(\frac{e_{1 j}^{3}}{a \varphi_{1 j} \varepsilon_{1 j}}\right) \\
x_{2 j c} & =A^{-1} n_{j c=} A^{-1}\left(-a \varphi_{1 j} \rho_{1 j}(\cdot) e_{1 j}-\widehat{D}_{1 j} \tanh \left(\frac{e_{1 j}^{3}}{a \varphi_{1 j} \varepsilon_{1 j}}\right)\right) .
\end{aligned}
$$

The design of outside loop controller $u$ is as follows:

$$
\begin{aligned}
C_{\varepsilon} & =(n \varepsilon)^{-(n / m n)}, \\
\varepsilon & =0.5 \\
\rho_{21 j}(\cdot) & =\frac{C_{\varepsilon}}{a \varphi_{1 j}}, \\
\rho_{2 j} & =\varsigma_{22} \frac{\Gamma^{3}}{4}\left(\left(\frac{\left\|\Psi_{2 j}\right\|}{\varphi_{2 j}}\right)^{4}+\left(\frac{1}{\varphi_{2 j}}\right)^{4}\right), \\
m_{j} & =-a \varphi_{2 j} e_{2 j}^{3}\left(\rho_{21 j}+\rho_{2 j}\right)-\widehat{D}_{2 j} \tanh \left(\frac{e_{2 j}}{a \varphi_{2 j} \varepsilon_{2 j}}\right), \\
u & =\frac{B^{T}}{B^{T} B}(M-f(x)) .
\end{aligned}
$$

Other relevant parameters are set as follows: $K=40, \varsigma_{1}=1,\left\|\Psi_{1}\right\|=2, a=1, \varsigma_{2}=1$, and $\left\|\Psi_{2}\right\|=2$. 
TABLE 1: Related parameters of the fixed-wing UAV.

\begin{tabular}{lcc}
\hline Variable & Implication & \\
\hline$V(\mathrm{~m} / \mathrm{s})$ & Velocity & Value \\
$m(\mathrm{~kg})$ & Weight & 10 \\
$b(\mathrm{~m})$ & Wingspan & 28 \\
$S\left(\mathrm{~m}^{2}\right)$ & Wing area & 3.1 \\
$\rho\left(\mathrm{kg} / \mathrm{m}^{3}\right)$ & Air density & 1.8 \\
\hline
\end{tabular}

TABLe 2: The initial values of the system states.

\begin{tabular}{lcc}
\hline Variable & Implication & Value \\
\hline$\vartheta(\mathrm{rad})$ & Pitch angle & 0.2 \\
$\psi(\mathrm{rad})$ & Yaw angle & 0.1 \\
$\gamma(\mathrm{rad})$ & Roll angle & 0.1 \\
$\omega_{x}(\mathrm{rad} / \mathrm{s})$ & Pitch rate & 0 \\
$\omega_{y}(\mathrm{rad} / \mathrm{s})$ & Yaw rate & 0 \\
$\omega_{z}(\mathrm{rad} / \mathrm{s})$ & Roll rate & 0 \\
$\widehat{D}(0)$ & Adaptive parameter & 0.001 \\
$\eta_{1}, \eta_{2}$ & Gain of the adaptive law & 1,2 \\
$\sigma_{1}, \sigma_{2}$ & Other parameters of the adaptive law & 5 \\
\hline
\end{tabular}

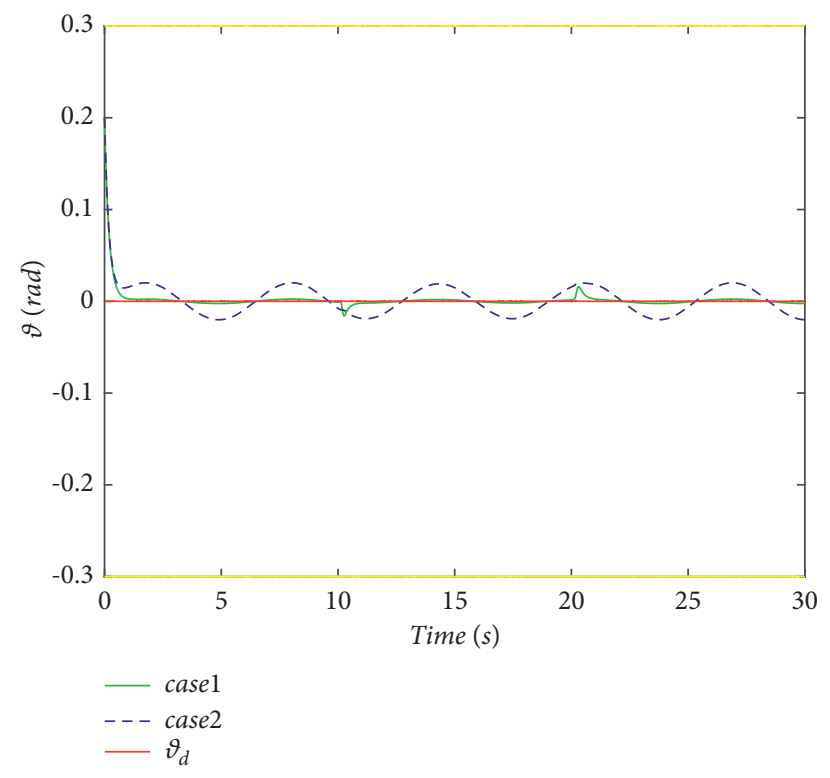

FIgURe 2: Variation curves of pitch angle in case 1 and case 2.

To show the advantages, the TAC (traditional adaptive control), DOBC, and general BLF are applied to the simulation.

Using the traditional adaptive method, it first applies backstepping design technology to constructively give the design steps of the state feedback adaptive controller and then combines Barbalat's lemma to analyze the stability of the closed-loop system, making the closed-loop system state globally asymptotically stable. The remaining closed-loop signals are uniformly bounded. The selected Lyapunov function is $V=0.5 e^{2}$. The relevant parameters are designed as $k_{1}=\operatorname{diag}\left[\begin{array}{lll}10 & 2 & 1\end{array}\right]$, and $k_{2}=\operatorname{diag}\left[\begin{array}{lll}10 & 2 & 1\end{array}\right]$.

DOBC (disturbance-observer-based control): by designing a disturbance observer to cancel the influence of disturbance on the servo system, the disturbance is regarded as a part of the system state, and the reduced-order state observer is used to estimate and cancel the disturbance. The design of the disturbance observer is $\Delta \widehat{C}=z+L x_{2}$ which is an auxiliary variable, and the relevant parameters are designed as $L=1, k_{1}=\operatorname{diag}\left[\begin{array}{lll}10 & 2 & 1\end{array}\right]$, and $k_{2}=\operatorname{diag}\left[\begin{array}{lll}10 & 2 & 1\end{array}\right]$.

Using the general BLF method, the symmetric positive definite log-type BLF is as follows:

$$
V_{i}=\frac{1}{4} \log \left(\frac{k_{b_{i}}^{4}}{k_{b_{i}}^{4}-e_{i}^{4}}\right)+\frac{\sigma_{i}}{2 \lambda_{i}} \widetilde{\mu}_{r, i}^{T} \widetilde{\mu}_{r, i} .
$$

It can guarantee that every step in the backstepping method does not violate the state constraint and 


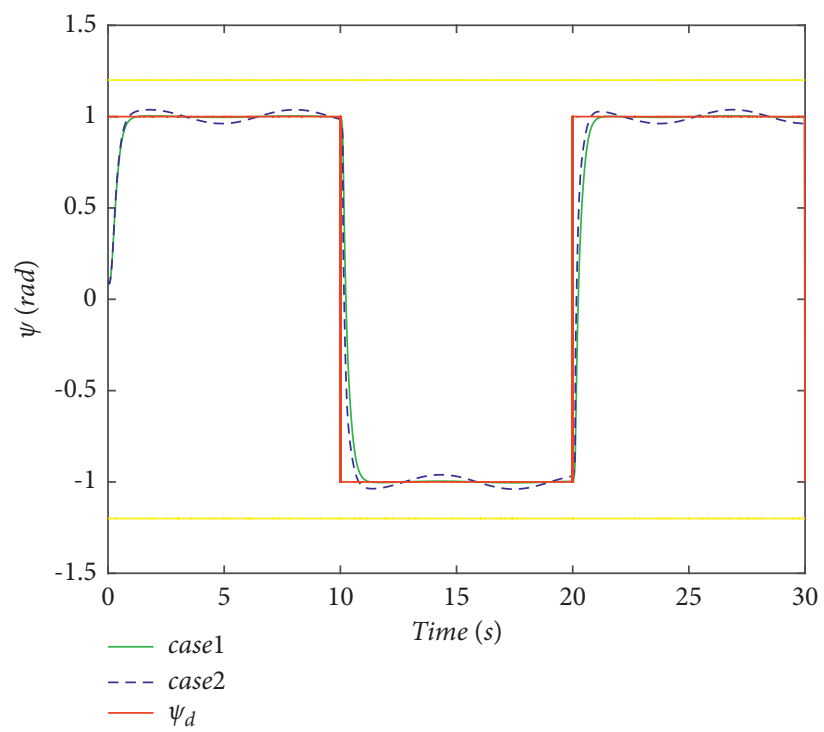

Figure 3: Variation curves of yaw angle in case 1 and case 2.

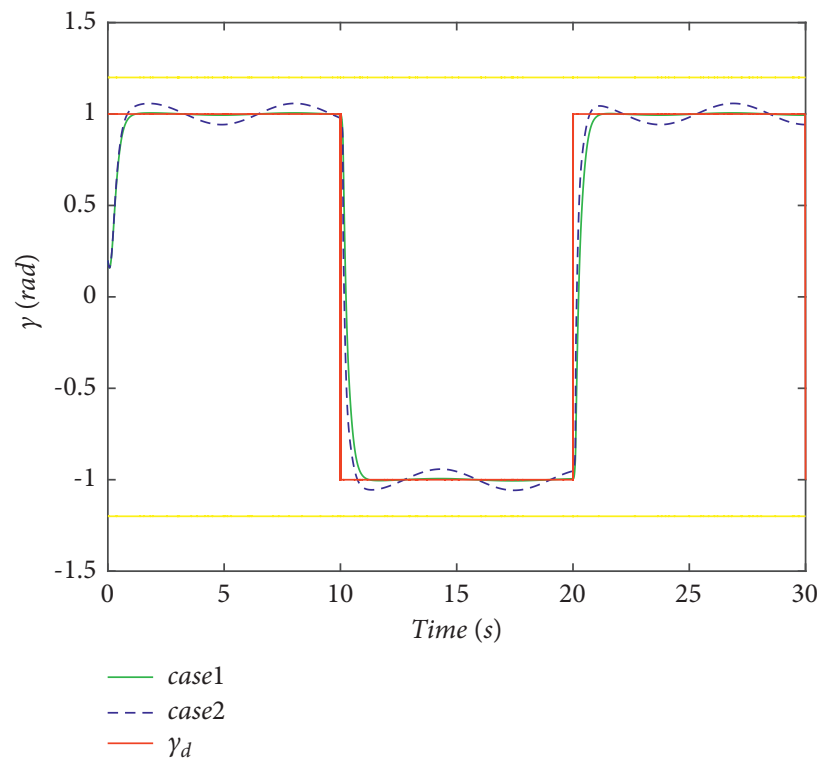

Figure 4: Variation curves of roll angle in case 1 and case 2.

guarantee the stability of the system, where $\sigma_{i}$ and $\lambda_{i}$ are positive design parameters, $k_{b i}=k_{c i}-\alpha_{i 0}$, and $u_{r, j}$ is an adaptive parameter. The relevant parameters are designed as $\gamma_{1}=0.5, k_{b 1}=1, k_{b 2}=2$, and $\gamma_{2}=0.2$.

HOBLF, DOBC, general adaptive, and general BLF were, respectively, used for simulation. The expected signal tracked was the square wave signal after second-order filtering, with an amplitude of 1 and a period of $20 \mathrm{~s}$.
The simulation results of the four methods are shown in Figures 6-11, where Figures 6 and 9 show the tracking trajectory and error curves of the pitch angle, respectively. Figures 7 and 10 show the yaw angle tracking track and error curve, respectively. Figures 8 and 11, respectively, show the tracking track and error curve of the roll angle. According to the analysis in Figures 6-8, the states in the traditional adaptive method and DOBC will exceed 

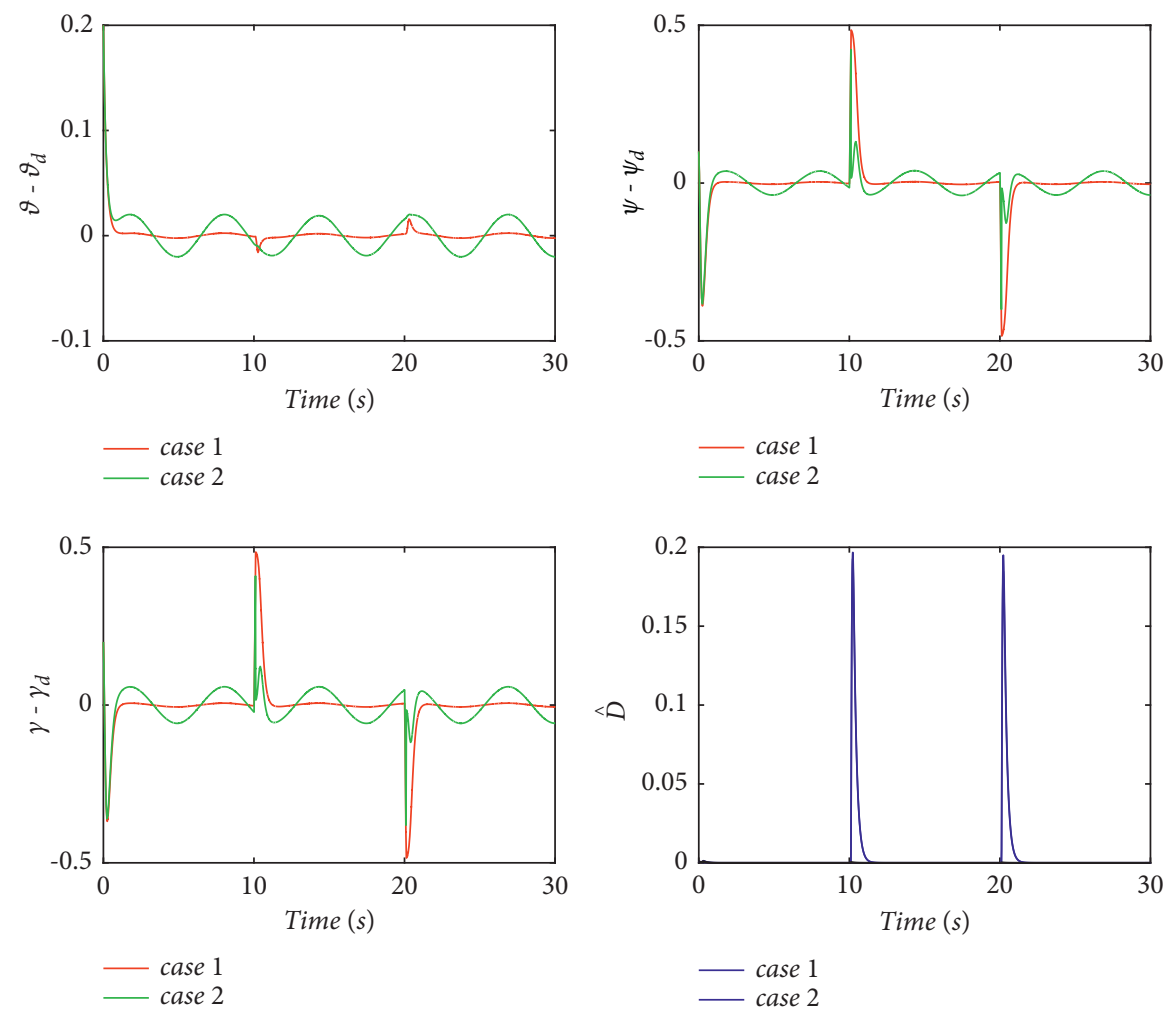

Figure 5: Tracking error of pitch angle, yaw angle, and roll angle in case 1 and case 2 and $\widehat{D}$ in case 1 and case 2 .

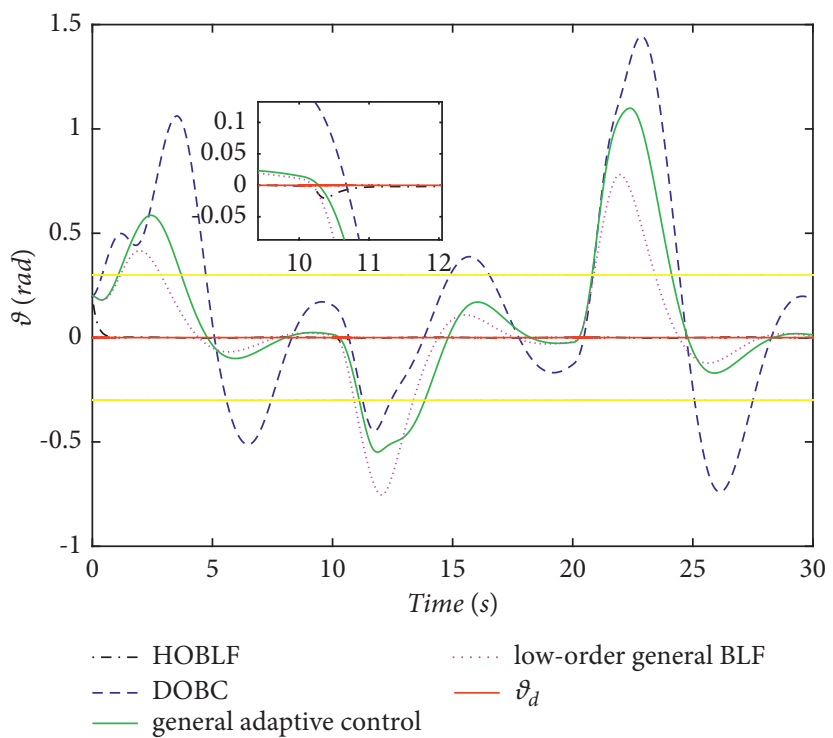

Figure 6: Tracking trajectory of pitch angle.

the state constraint and have a large deviation. The tracking trajectory of the high-order BLF and general BLF in three directions is always between the upper and lower limits of the state constraint. However, for the tracking with a constant expected signal, high-order BLF is more stable and has less error. It can be easily known from Figures 9-11 that compared with the high-order BLF method, the traditional adaptive method, DOBC, and general BLF have a larger tracking trajectory and error. 


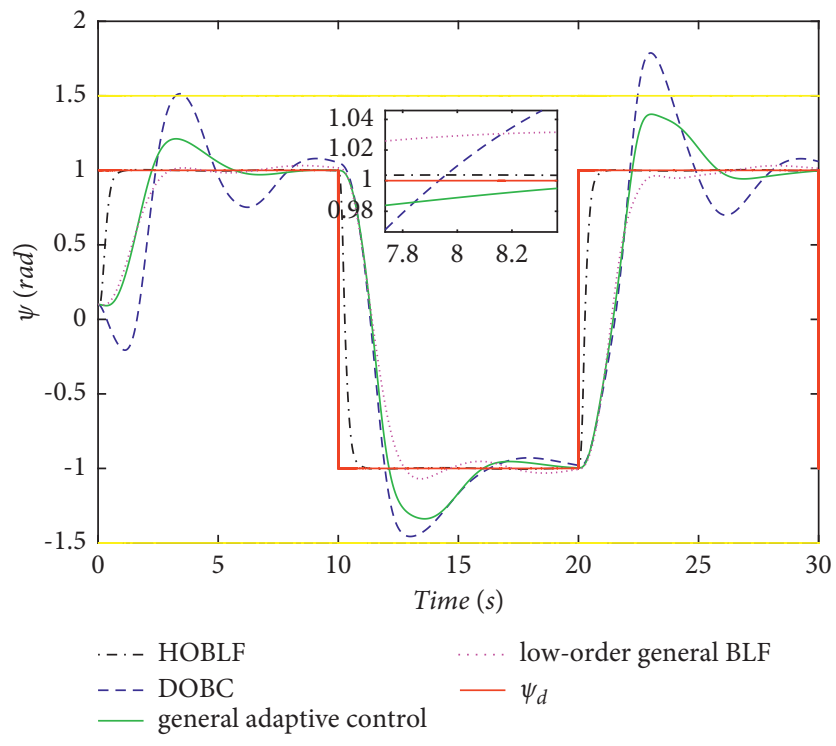

FIgURE 7: Tracking trajectory of yaw angle.

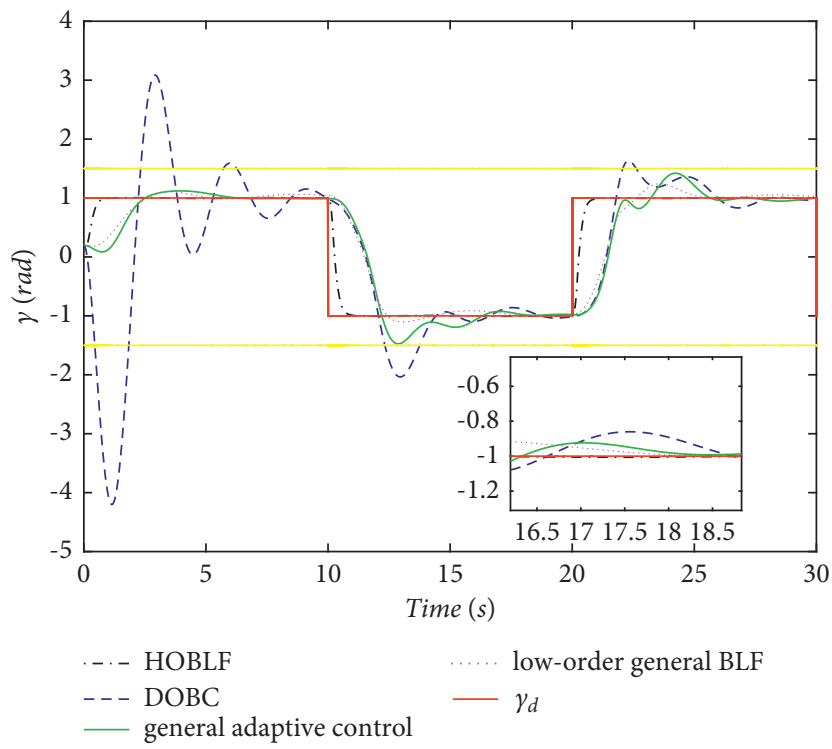

FIgURE 8: Tracking trajectory of roll angle. 


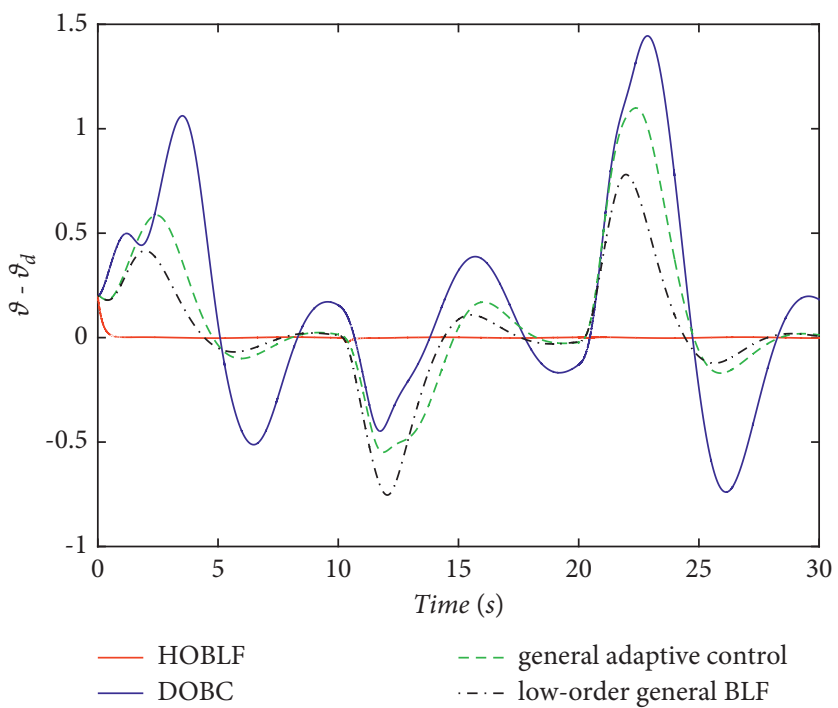

FIgURE 9: Tracking error of pitch angle.

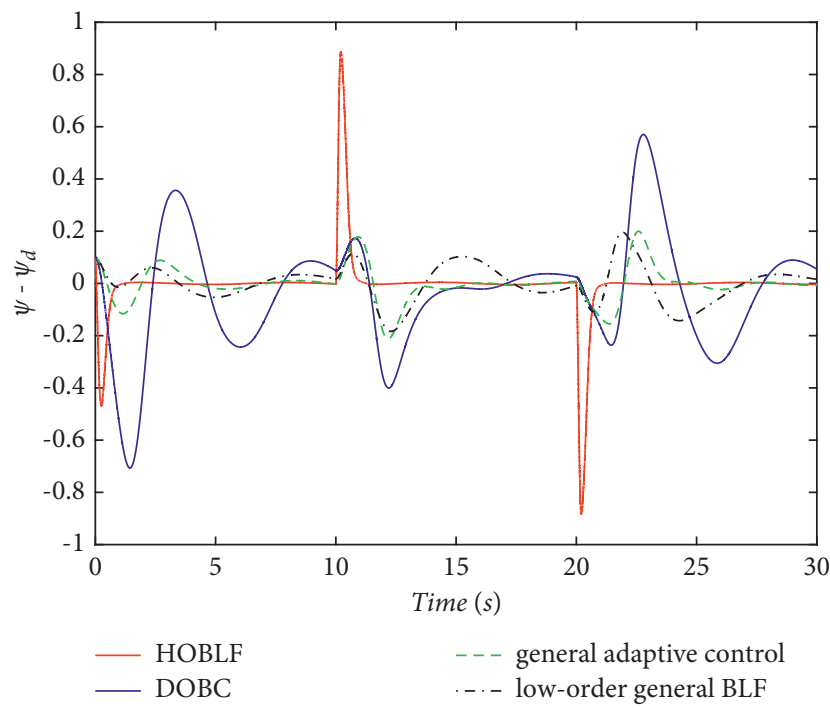

FIgURE 10: Tracking error of yaw angle.

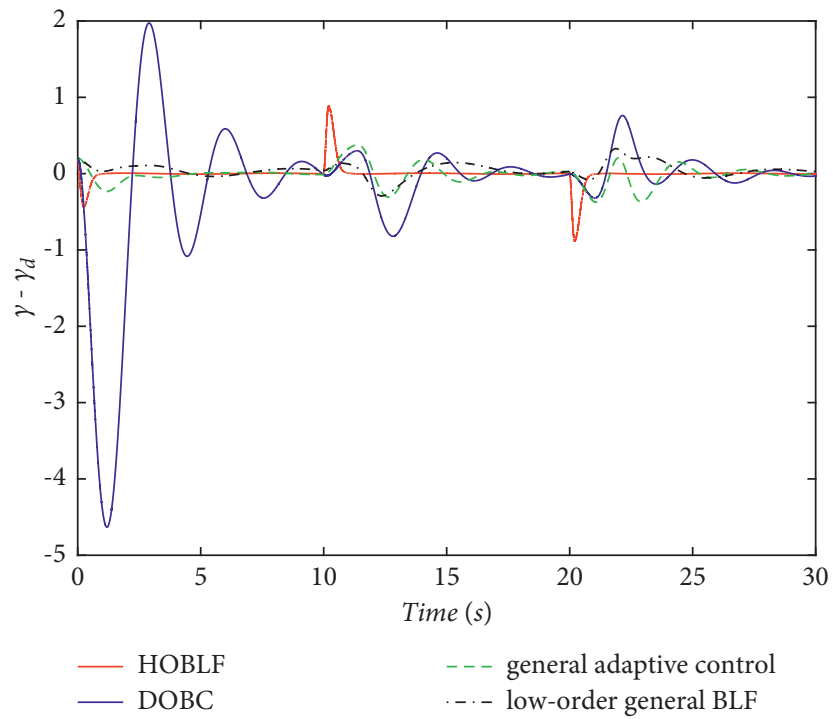

FIgURE 11: Tracking error of roll angle. 


\section{Conclusion}

In this work, an adaptive fuzzy controller has been proposed for a fixed-wing UAV control system of fullstate constraints and unknown external disturbances. The high-order BLF (HOBLF) is firstly introduced for the UAV systems to realize the state constraints. Meanwhile, the unknown nonlinear functions have been compensated by the fuzzy system and backstepping control method to ensure the stability of the system. According to simulation results and comparison, we have made the conclusion that the proposed control is able to track a desired trajectory with an excellent performance without violating the state constraints. Moreover, further research based on the current results of this paper is how to verify the control feasibility of the UAV system with existing time-varying state constraint issues.

\section{Data Availability}

The data used to support the findings of this study are included within the article.

\section{Conflicts of Interest}

The authors declare that they have no conflicts of interest.

\section{Acknowledgments}

This work was supported in part by the Basic Research Program of Natural Science of Shaanxi Province (Grant no. 2019ZY-CXPT-03-02).

\section{References}

[1] L. Fu, F. Xie, D. Wang, and G. Meng, "The overview for UAV air-combat decision method," in Proceedings of the 26th Chinese Control and Decision Conference (2014 CCDC), pp. 3380-3384, Changsha, China, June 2014.

[2] M. Liu, G. K. Egan, and F. Santoso, "Modeling, autopilot design, and field tuning of a UAV with minimum control surfaces," IEEE Transactions on Control Systems Technology, vol. 23, no. 6, pp. 2353-2360, 2015.

[3] L. N. Fei Ailing and S. Li, "Auto-disturbance and anti-step control of fixed-wing UAV," Control Theory \& Applications, vol. 33, no. 10, 2016.

[4] Y. Wang, X. Zhu, Z. Zhou, M. Xu, and Y. Feng, "Control method of flying-wing layout drone passing through microdowndraft," Acta Aeronautica et Astronautica Sinica, vol. 1, pp. 6-8, 2003.

[5] D. Q. Zhang Chaofan, "Adaptive sliding mode control of fixed-wing UAV considering input saturation," Journal of Aeronautics, vol. 41, no. S1, Article ID 723755, 2020.

[6] Y. Liu, S. Rajappa, J. M. Montenbruck et al., "Robust nonlinear control approach to nontrivial maneuvers and obstacle avoidance for quadrotor UAV under disturbances," Robotics and Autonomous Systems, vol. 98, pp. 317-332, 2017.

[7] W. MacKunis, Z. D. Wilcox, M. K. Kaiser, and W. E. Dixon, "Global adaptive output feedback tracking control of an unmanned aerial vehicle," IEEE Transactions on Control Systems Technology, vol. 18, no. 6, pp. 1390-1397, 2010.
[8] S. Kurnaz, O. Cetin, and O. Kaynak, "Adaptive neuro-fuzzy inference system based autonomous flight control of unmanned air vehicles," Expert Systems with Applications, vol. 37, no. 2, pp. 1229-1234, 2010.

[9] L. Zhi and W. Yong, "Fuzzy adaptive tracking control within the full envelope for an unmanned aerial vehicle," Chinese Journal of Aeronautics, vol. 27, no. 5, pp. 1273-1287, 2014.

[10] M. Golestani, S. Mobayen, and H. Richter, "Fast robust adaptive tracker for uncertain nonlinear second-order systems with time-varying uncertainties and unknown parameters," International Journal of Adaptive Control and Signal Processing, vol. 32, no. 12, pp. 1764-1781, 2018.

[11] S. Mobayen, J. Ma, G. Pujol-Vazquez, L. Acho, and Q. Zhu, "Adaptive finite-time stabilization of chaotic flow with a single unstable node using a nonlinear function-based global sliding mode," Iranian Journal of Science and Technology, Transactions of Electrical Engineering, vol. 43, pp. S339-S347, 2019.

[12] S. Mobayen, "Adaptive global sliding mode control of underactuated systems using a super-twisting scheme: an experimental study," Journal of Vibration and Control, vol. 25, no. 16, pp. 2215-2224, 2019.

[13] A. Abdessameud and A. Tayebi, "Global trajectory tracking control of VTOL-UAVs without linear velocity measurements," Automatica, vol. 46, no. 6, pp. 1053-1059, 2010.

[14] K. Natesan, D. W. Gu, I. Postlethwaite, and J. Chen, "Design of flight controllers based on simplified LPV model of a UAV," in Proceedings of the 45th IEEE Conference on Decision and Control, pp. 37-42, San Diego, CA, USA, December 2006.

[15] Y. Yeonsik Kang and J. K. Hedrick, "Linear tracking for a fixed-wing UAV using nonlinear model predictive control," IEEE Transactions on Control Systems Technology, vol. 17, no. 5, pp. 1202-1210, 2009.

[16] X. Ruan and X. Hou, "Nonlinear predictive decoupling control of rotorcraft UAV using serial decoupling method," Applied Mechanics and Materials, vol. 241-244, pp. 1240-1247, 2013.

[17] S. Akyurek, U. Kaynak, and C. Kasnakoglu, "Altitude control for small fixed-wing aircraft using Ho loop-shaping method," IFAC-PapersOnLine, vol. 49, no. 9, pp. 111-116, 2016.

[18] D. Rotondo, A. Cristofaro, K. Gryte, and T. A. Johansen, "LPV model reference control for fixed-wing $\mathrm{UAVs}^{* *} \mathrm{D}$. Rotondo acknowledges that this work was carried out during the tenure of an ERCIM Alain Bensoussan Fellowship Programme. This work was supported by the Research Council of Norway through the Centers of Excellence funding scheme, Project number 223254 - centre for Autonomous Marine Operations and Systems (NTNU-AMOS), and project 261791," IFACPapersOnLine, vol. 50, no. 1, pp. 11559-11564, 2017.

[19] J. Chen, R. Sun, and B. Zhu, "Disturbance observer-based control for small nonlinear UAV systems with transient performance constraint," Aerospace Science and Technology, vol. 105, Article ID 106028, 2020.

[20] Y. Yu, H. Wang, X. Shao, and Y. Huang, "The Attitude control of UAV in carrier landing based on ADRC," in Proceedings of the 2016 IEEE Chinese Guidance, Navigation and Control Conference (CGNCC), pp. 832-837, Nanjing, China, August 2017.

[21] C. Linqi and L. Tinghui, "UAV control system based on backstepping and active disturbance rejection control," in Proceedings of the 2018 3rd Joint International Information Technology, Mechanical and Electronic Engineering Conference (JIMEC 2018), Chongqing, China, December 2018.

[22] J. Mullen, S. C. C. Bailey, and J. B. Hoagg, "Filtered dynamic inversion for altitude control of fixed-wing unmanned air 
vehicles," Aerospace Science and Technology, vol. 54, pp. 241-252, 2016.

[23] H. Castañeda, O. S. Salas-Peña, and J. d. León-Morales, "Extended observer based on adaptive second order sliding mode control for a fixed wing UAV," ISA Transactions, vol. 66, pp. 226-232, 2017.

[24] J. Tan and S. Guo, "Backstepping control with fixed-time prescribed performance for fixed wing UAV under model uncertainties and external disturbances," International Journal of Control, pp. 1-18, 2020.

[25] C. Fu, Y. Tian, H. Huang, L. Zhang, and C. Peng, "Finite-time trajectory tracking control for a 12-rotor unmanned aerial vehicle with input saturation," ISA Transactions, vol. 81, pp. 52-62, 2018.

[26] H. Wang, W. Bai, and P. X. Liu, "Finite-time adaptive faulttolerant control for nonlinear systems with multiple faults," IEEE/CAA Journal of Automatica Sinica, vol. 6, no. 6, pp. 1417-1427, 2019.

[27] M. Chen, H. Wang, and X. Liu, "Adaptive fuzzy practical fixed-time tracking control of nonlinear systems," IEEE Transactions on Fuzzy Systems, vol. 29, no. 3, pp. 664-673, 2021.

[28] D.-J. Li and D.-P. Li, “Adaptive controller design-based neural networks for output constraint continuous stirred tank reactor," Neurocomputing, vol. 153, pp. 159-163, 2015.

[29] W. He, S. Zhang, and S. S. Ge, "Adaptive control of a flexible crane system with the boundary output constraint," IEEE Transactions on Industrial Electronics, vol. 61, no. 8, pp. 4126-4133, 2014.

[30] W. He, Y. Chen, and Z. Yin, "Adaptive neural network control of an uncertain robot with full-state constraints," IEEE Transactions on Cybernetics, vol. 46, no. 3, pp. 620-629, 2016.

[31] A. Bemporad, "Reference governor for constrained nonlinear systems," IEEE Transactions on Automatic Control, vol. 43, no. 3, pp. 415-419, 1998.

[32] D. Q. Mayne, J. B. Rawlings, C. V. Rao, and P. O. M. Scokaert, "Constrained model predictive control: stability and optimality," Automatica, vol. 36, no. 6, pp. 789-814, 2000.

[33] D. Dehaan and M. Guay, "Extremum-seeking control of stateconstrained nonlinear systems," IFAC Proceedings Volumes, vol. 37, no. 13, pp. 663-668, 2004.

[34] T. Tingshu $\mathrm{Hu}$ and Z. Zongli Lin, "Composite quadratic lyapunov functions for constrained control systems," IEEE Transactions on Automatic Control, vol. 48, no. 3, pp. 440450, 2003.

[35] T. Hu, Z. Lin, and B. M. Chen, "An analysis and design method for linear systems subject to actuator saturation and disturbance," Automatica, vol. 38, no. 2, pp. 351-359, 2002.

[36] K. B. Ngo, R. Mahony, and Z. P. Jiang, "Integrator backstepping using barrier functions for systems with multiple state constraints," in Proceedings of the 44th IEEE Conference on Decision and Control, and the European Control, pp. 8306-8312, Seville, Spain, December 2005.

[37] K. P. Tee and S. S. Ge, "Control of nonlinear systems with partial state constraints using a barrier Lyapunov function," International Journal of Control, vol. 84, no. 12, pp. 20082023, 2011.

[38] Y.-J. Liu and S. Tong, "Barrier Lyapunov functions-based adaptive control for a class of nonlinear pure-feedback systems with full state constraints," Automatica, vol. 64, pp. 70-75, 2016.

[39] Y.-J. Liu, S. Lu, S. Tong, X. Chen, C. L. P. Chen, and D.-J. Li, "Adaptive control-based barrier lyapunov functions for a class of stochastic nonlinear systems with full state constraints," Automatica, vol. 87, pp. 83-93, 2018.

[40] Y.-J. Liu, S. Tong, C. L. P. Chen, and D.-J. Li, “Adaptive NN control using integral barrier lyapunov functionals for uncertain nonlinear block-triangular constraint systems," IEEE Transactions on Cybernetics, vol. 47, no. 11, pp. 3747-3757, 2017.

[41] K. P. Tee, B. Ren, and S. S. Ge, "Control of nonlinear systems with time-varying output constraints," Automatica, vol. 47, no. 11, pp. 2511-2516, 2011.

[42] K. P. Tee, S. S. Ge, and E. H. Tay, "Output-feedback adaptive control of electrostatic microactuators," Proceedings-American Control Conference, vol. 17, no. 2, pp. 4215-4220, 2009.

[43] B. Xu, Z. Shi, F. Sun, and W. He, "Barrier Lyapunov function based learning control of hypersonic flight vehicle with AOA constraint and actuator faults," IEEE Transactions on Cybernetics, vol. 49, no. 3, pp. 1047-1057, 2019.

[44] C. Dong, Y. Liu, and Q. Wang, "Barrier lyapunov function based adaptive finite-time control for hypersonic flight vehicles with state constraints," ISA Transactions, vol. 96, pp. 163-176, 2020.

[45] W. Sun, S.-F. Su, G. Dong, W. Bai, and V.-T. Nguyen, "Reduced adaptive fuzzy tracking control for high-order stochastic nonstrict feedback nonlinear system with full-state constraints," IEEE Transactions on Systems, Man, and Cybernetics: Systems, vol. 51, no. 3, pp. 1-11, 2021.

[46] Y. Tang, D. Lin, L. Cao, and Y. Liu, "Attitude control of fixedwing UAV under model uncertainty and disturbances," Electronics Optics and Control, vol. 1, no. 27, pp. 85-89, 2020. 